\title{
Estimation in Cellular Radio Systems
}

\author{
Jonas Blom, Fredrik Gunnarsson and Fredrik Gustafsson \\ Department of Electrical Engineering \\ Linköpings universitet, SE-581 83 Linköping, Sweden \\ WWW: http://www. control.isy.liu.se \\ Email: jb@isy.liu.se, fred@isy.liu.se, \\ fredrik@isy.liu.se
}

April 16, 1999

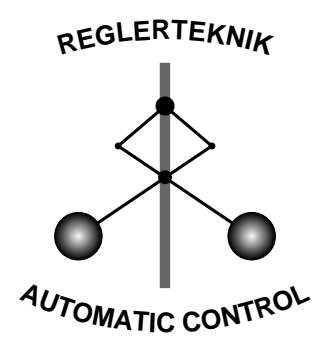

LINKÖPING

Report no.: LiTH-ISY-R-2145

Submitted to ICASSP'99, Phoenix, AZ, USA

Technical reports from the Automatic Control group in Linkping are available by anonymous ftp at the address ftp.control.isy.liu.se. This report is contained in the compressed postscript file 2145.ps. Z. 



\begin{abstract}
The problem to track time-varying parameters in cellular radio systems is studied, and the focus is on estimation based only on the signals that are readily available. Previous work have demonstrated very good performance, but were relying on analog measurement that are not available. Most of the information is lost due to quantization and sampling at a rate that might be as low as $2 \mathrm{~Hz}$ (GSM case). For that matter a Maximum Likelihood estimator have been designed and exemplified in the case of GSM. Simulations indicate good performance both when most parameters are varying slowly, and when subject to fast variations as in realistic cases. Since most computations take place in the base stations, the estimator is ready for implementation in a second generation wireless system. No update of the software in the mobile stations is needed.
\end{abstract}

\title{
Keywords: Maximum Likelihood Estimation; Point-mass approx- imation; Cellular radio systems; GSM; Measurement reports
}




\section{Introduction}

Due to the rapid expansion of the wireless mobile market, and the need for wideband multimedia services, the available bandwidth has to be better utilized. Several transmitter power control algorithms have been proposed to improve the capacity. Most schemes strive to balance the carrier-to-interference ratios (C/I) on each channel such that every mobile or base station achieve the same $\mathrm{C} / \mathrm{I}$ [10].

To avoid extensive signaling in the network, it is desirable to use distributed algorithms, where the transmitter powers are locally controlled based on local measurements or estimates. For an overview, see $[3,4]$ and the references therein. Most algorithms are relying on accurate $\mathrm{C} / \mathrm{I}$ estimates, and popular methods for estimation with good performance are described in $[2,9]$. In those approaches, however, it is assumed that analog signal strength measurements are available, which is not the case in a real system. Instead the information is regularly available in measurement reports. One of the core problems is to locally extract as much relevant information as possible from these reports. This includes the carrier signal and the distribution of the interference.

It is common that the measurement reports are comprising coarsely quantized values reflecting the perceived quality (Quality Indicator, QI) and signal strength (Received Signal Strength Indicator, RSSI). These values are depending on the parameters to be estimated. Hence, the measurement reports contain information from two conceptually different information sources, which leads to a sensor fusion formulation of the problem.

\section{System Model}

Signal gains and power levels can be expressed using different scales. Logarithmic (e.g. $d B$ or $d B m$ ) or linear are often used. To avoid confusion we will employ the convention of indicating linearly scaled values with a bar. Thus $\bar{g}_{i j}$ is a value in linear scale, and $g_{i j}$ the corresponding value in logarithmic scale.

Assume that the $m$ mobile stations on a specific radio channel are transmitting using the powers $p_{i}(t)$, where $i=1, \ldots, m$. The signal between mobile station $i$ and base station $j$ is attenuated by the signal gain $g_{i j}(t)(<0)$. Thus the corresponding connected base station will experience a desired carrier signal $C_{i}(t)=p_{i}(t)+g_{i i}(t)$ and an interference plus noise $I_{i}(t)$

$$
I_{i}(t)=10 \log _{10}\left(\sum_{j \neq i} \bar{g}_{i j}(t) \bar{p}_{j}(t)+\bar{\nu}_{i}(t)\right),
$$

where $\bar{\nu}_{i}(t)$ denotes the thermal noise. The $\mathrm{C} / \mathrm{I}$ at base station $i$ is defined by

$$
\gamma_{i}(t)=p_{i}(t)+g_{i i}(t)-I_{i}(t) .
$$

In the rest of the article, only the situation at reciever $i$ will be considered. Therefore, the $i$ index will be dropped for simplicity. 
The interference can be viewed as a stochastic variable with a distribution parametrized by

$$
m_{I}, \theta_{1}, \ldots, \theta_{n}
$$

where $m_{I}$ is the mean value. Estimators used in previous work are only characterizing the interference by its mean value, see e.g. [1], and therefore this approach describes the interference more thoroughly. Thus the parameters to be estimated are given by

$$
\boldsymbol{\theta}=\left[C, m_{I}, \theta_{1}, \ldots, \theta_{n}\right]^{T} .
$$

The available measurements at the receiver in focus $\boldsymbol{x}=\left[x^{(1)}, x^{(2)}\right]^{T}$ can thus be expressed by

$$
\begin{aligned}
\mathrm{RSSI}: x^{(1)} & =h_{1}\left(\bar{C}, \bar{m}_{I}, \theta_{1}, \ldots, \theta_{n}\right) \\
\mathrm{QI}: x^{(2)} & =h_{2}\left(\bar{C}, \bar{m}_{I}, \theta_{1}, \ldots, \theta_{n}\right),
\end{aligned}
$$

where the functions $h_{k}(\cdot)$ return realizations of stochastic variables.

\subsection{Example: Frequency Hopping GSM}

In order to characterize the interference distribution, a simulation model was used. It was assumed that the interference is constant during a burst (corresponding to $0.577 \mathrm{~ms}$ ). The gains of the transmitted powers, $g_{i j}$, were modeled by the distance depending path loss and fading (shadow and Rayleigh). For further details regarding propagation modeling, we refer to [8]. The simulations indicate that the interference experienced by a user in the network is approximately normal distributed $N\left(m_{I}, \sigma_{I}\right)$. Furthermore, we note that this conclusion is relatively independent not only of network specific parameters such as cell radius, but also of the distribution of the transmitted powers. This is also supported by theoretical results, see $[8,7]$.

In GSM the measurement reports consist of RXLEV and RXQUAL. RXLEV is a signal strength measure, which has been quantized in 64 levels, and RXQUAL is a logarithmic measure of the bit error probability, quantized in 8 levels.

In the GSM case we thus have the following measurements and parameters.

$$
\begin{aligned}
\boldsymbol{x} & =(\mathrm{RXLEV}, \mathrm{RXQUAL})^{T} \\
\boldsymbol{\theta} & =\left(C, m_{I}, \sigma_{I}\right)^{T} .
\end{aligned}
$$

\section{Maximum Likelihood Estimation}

The estimator can be constructed in numerous ways, but we have chosen a Maximum Likelihood (ML) estimator, since it is successfully enables this data fusion and is an implementationally simple algorithm. 
The method of ML estimation is based on a simple idea. Different probability density functions generate different data samples and any given data sample is more likely to have come from a particular distribution than from others. We will not go into the basic details of how ML estimation is implemented, instead, we refer the reader who is not familiar with these concepts to [6].

Let $\boldsymbol{x}_{t}$ denote a measurement at time $t$, and $\boldsymbol{\theta}$ the parameters describing the interference distribution together with the carrier. The probability function of a single measurement is a product of two probability functions; one for the RSSI and one for the QI as below.

$$
f_{X}\left(\boldsymbol{x}_{t} ; \boldsymbol{\theta}\right)=f_{1}\left(\boldsymbol{x}_{t} ; \boldsymbol{\theta}\right) \cdot f_{2}\left(\boldsymbol{x}_{t} ; \boldsymbol{\theta}\right)
$$

Since the parameters we want to estimate are time varying, we want the ML estimation procedure to be adaptive. In order to accomplish this, a forgetting factor, $\lambda$, is introduced. Then the likelihood function is given by the following recursion

$$
l_{t}(\boldsymbol{\theta})=f_{X}\left(\boldsymbol{x}_{t} ; \boldsymbol{\theta}\right)^{1-\lambda} \cdot l_{t-1}(\boldsymbol{\theta})^{\lambda}
$$

Note that if the likelihood is equal to zero for values in the parameter space at some time, it will remain zero. This is not desirable, and therefore a threshold $f_{\min }$ is introduced. Furthermore, we use the logarithm of the likelihood to avoid numerical problems.

The estimate, $\hat{\boldsymbol{\theta}}$ is then obtained as the values which maximize the likelihood function. In order to obtain smooth estimates, it is preferable to filter each of the estimates separately, since they change at different rates. Exponential filtering is suitable for this purpose, see $[3,5]$.

Thus, we propose the following algorithm for the estimator:

\section{Algorithm: ML Estimation}

Let the measurements at time $t$, be given by the vector $\boldsymbol{x}_{t}$, with the corresponding probability function $f_{X}\left(\boldsymbol{x}_{t} ; \boldsymbol{\theta}\right)$. Define $g_{X}\left(\boldsymbol{x}_{t} ; \boldsymbol{\theta}\right)$ by

$$
g_{X}\left(\boldsymbol{x}_{t} ; \boldsymbol{\theta}\right)=\max \left\{f_{X}\left(\boldsymbol{x}_{t} ; \boldsymbol{\theta}\right), f_{\min }\right\} .
$$

Update the likelihood function according to

$$
\log l_{t}(\boldsymbol{\theta})=(1-\lambda) \log g_{X}\left(\boldsymbol{x}_{t} ; \boldsymbol{\theta}\right)+\lambda \log l_{t-1}(\boldsymbol{\theta}),
$$

with the intial likelihood $l_{0}(\boldsymbol{\theta})$ equal to a Gaussian pdf. The estimate $\boldsymbol{\theta}(t)$ is given by

$$
\hat{\boldsymbol{\theta}}^{M L}(t)=\underset{\boldsymbol{\theta}}{\arg \max } l_{t}(\boldsymbol{\theta}) .
$$

Post-filter the estimates separately as

$$
\hat{\theta}_{i}(t+1)=\left(1-\mu_{i}\right) \hat{\theta}_{i}^{M L}(t+1)+\mu_{i} \hat{\theta}_{i}(t)
$$




\subsection{Example: Frequency Hopping GSM}

The ML estimation algorithm requires the probability functions of the measurements RXQUAL and RXLEV. In general it is not possible to derive analytical expressions. Instead we compute these functions in a grid covering the interesting parameter space. Based on models of the coding, modulation and interfaces according to the GSM standard and implementation, the value in each grid point can be approximated using Monte-Carlo simulations. The approximation of the RXQUAL probability function is illustrated in Figure 1.
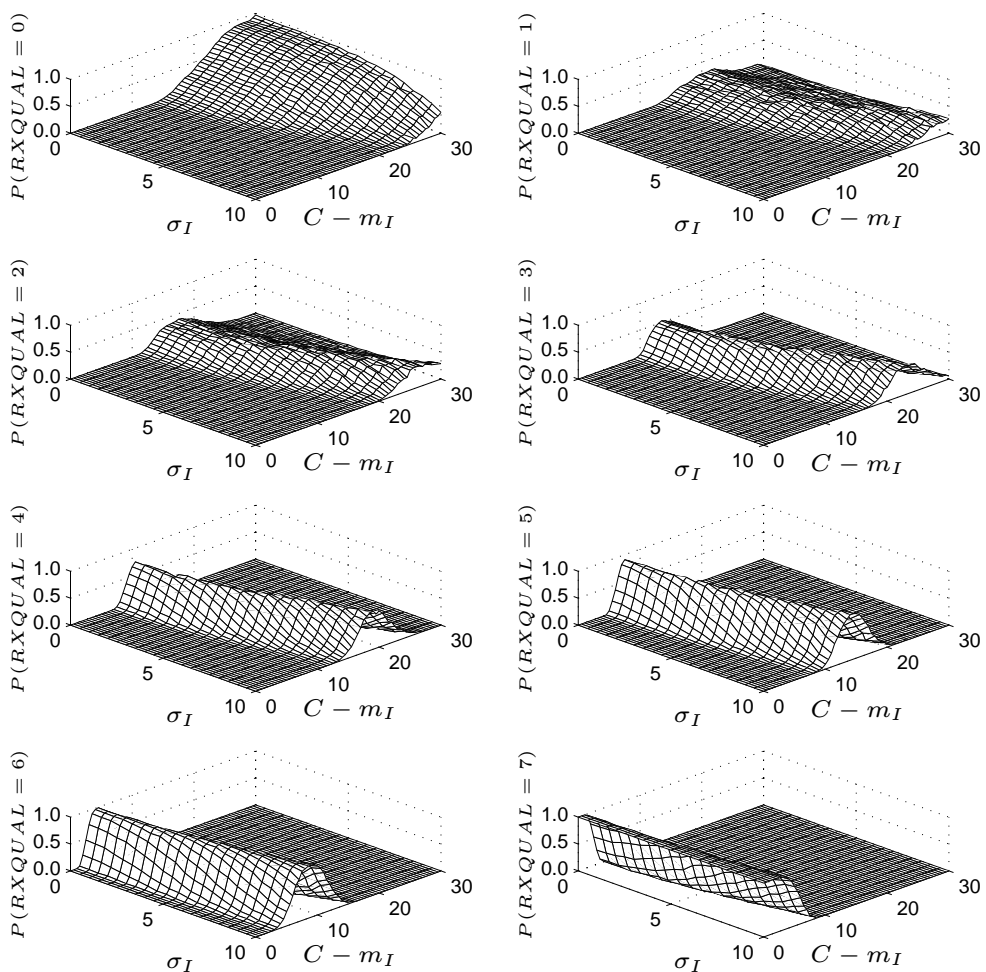

Figure 1: The RXQUAL probability function.

\section{Simulations}

The simulation environment is GSM specific and the interfaces are the same as in the real systems. The simulator parameters are summarized in Table 1.

In order to get a feel for ML estimation, we first consider a simple case. All parameters are fixed except $m_{I}$ which changes abruptly. The simulator is used to generate a sequence of measurement reports, which are fed to the estimator. The results are found in Figure 2, where we see that the estimator is able to track the parameters. The operation of the estimator in this case is further 


\begin{tabular}{ll}
\hline Frequency band & $900 \mathrm{MHz}$ \\
Antennas & Sectorized \\
Cell radius & $1000 \mathrm{~m}$ \\
Cell layout & $5 \times 5$ clusters of 9 cells \\
Frequency hopping & Pseudo-random \\
Control sample interval & $T_{c}=0.48 \mathrm{~s}$ \\
Burst time & $0.577 \mathrm{~ms}$ \\
Mobile station & $\mathrm{GSM}$ class 4 \\
Mean mobile station speed & $50 \mathrm{~km} / \mathrm{h}$ \\
\hline
\end{tabular}

Table 1: System simulation parameters.

illuminated by Figure 3 .

a.

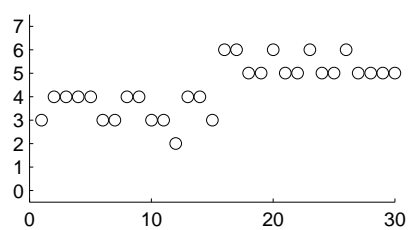

c.

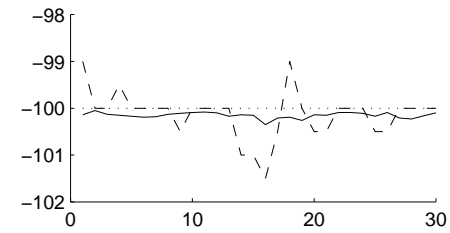

e.

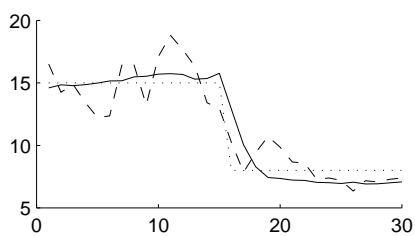

b.

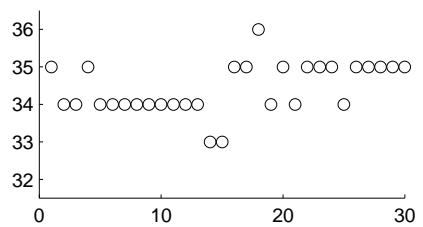

d.

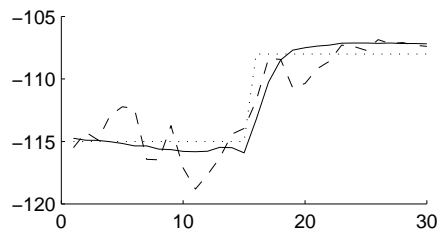

f.

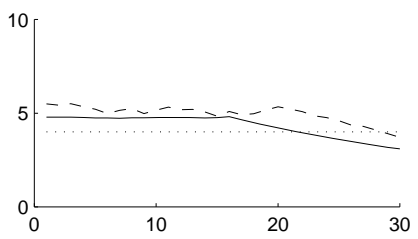

Figure 2: Estimation when there is an abrupt change in the true $m_{I}$. Above are the estimated values (dashed) plotted together with an average over several estimations (solid) and the true values (dotted). Measurement reports: a) RXQUAL and b) RXLEV. Estimated parameters: c) Carrier, $C$, d) Mean interference, $m_{I}$, e) C/I, $C-m_{I}$, f) Std.dev., $\sigma_{I}$.

The normal situation is that the carrier $C$ and mean interference $m_{I}$ are subject to fast variations due to shadow and multipath fading, see [8]. The measurement reports in Figure 4 are obtained from network simulations. These are fed to the estimator and the estimated parameters are compared to the true values, see Figure 5. Despite the loss of information in the quantization, the estimates are reasonably accurate. 

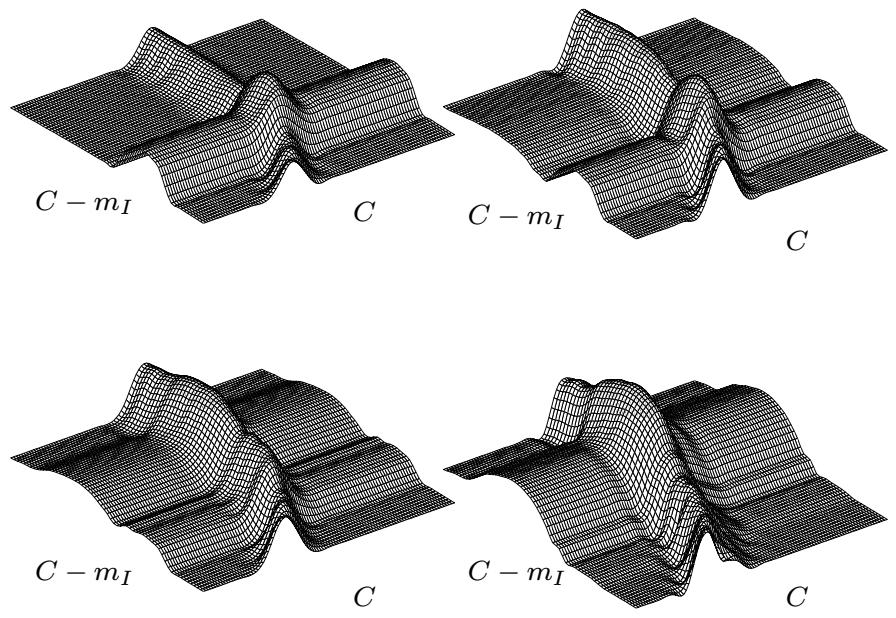

Figure 3: The shape and peak position of the likelihood function changes, when the value of $m_{I}$ changed abruptly. The maximum of this function is seen to move in the $\left(C-m_{I}\right)$-direction. The $\sigma$ direction has been eliminated by selecting the true value.

\section{Conclusions}

In this work we have focused on estimation in cellular radio systems based only on the signals that are readily available. Previous work have demonstrated very good performance, but were relying on analog measurement that are not available. Most of the information is lost due to quantization and sampling at a rate that might be as low as $2 \mathrm{~Hz}$ (GSM case).

A Maximum Likelihood estimator has been designed and exemplified in the case of GSM. Simulations indicate good performance both when most parameters are varying slowly, and when subject to fast variations as in realistic cases.

It is ready for implementation in a second generation wireless system. The only component that needs to be updated is the software in the base stations, where the output powers are computed. However, this estimation method is general, and will be useful in a third generation wireless system as well. 
a.

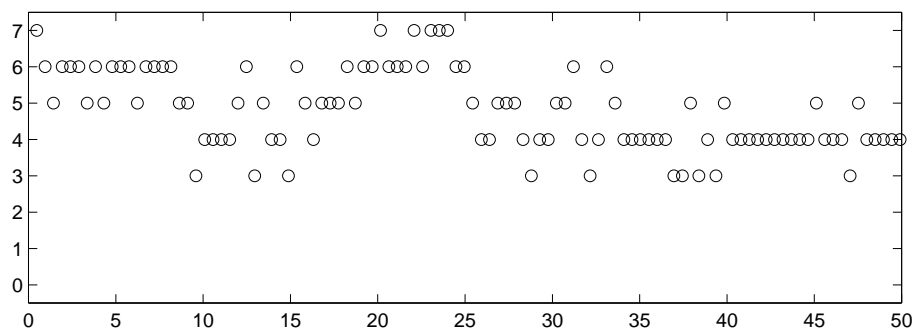

b.

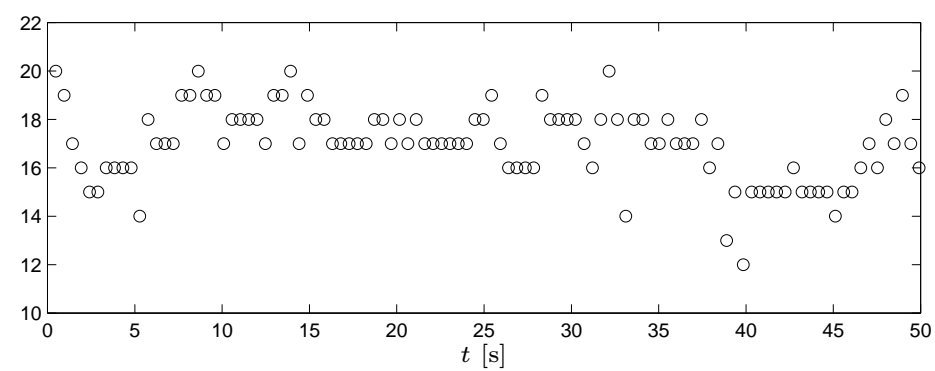

Figure 4: Measurement reports, consisting of a) RXQUAL and b) RXLEV, describing the perceived quality and signal strength respectively. These values are fed to the estimator.

a.

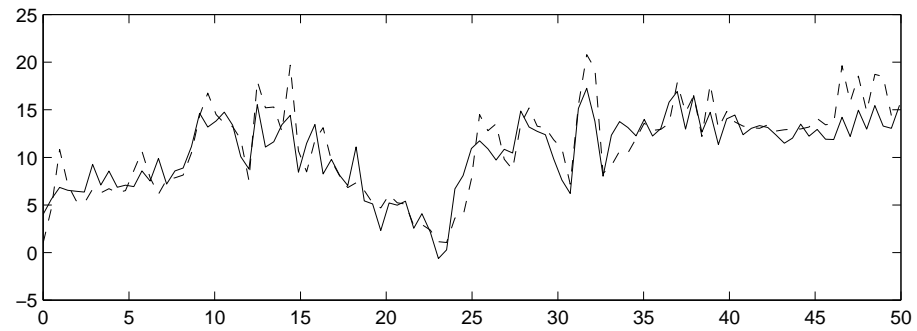

b.

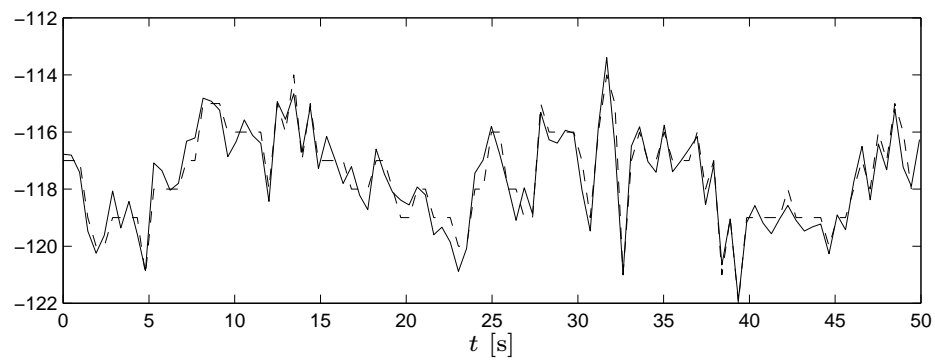

Figure 5: Given the measurement reports in Figure 4 the estimator extracts e.g. a) C/I and b) Carrier. The estimated values (solid) are compared to the true ones (dashed). 


\section{References}

[1] M. Almgren, H. Andersson, and K. Wallstedt. Power control in a cellular system. In Proc. IEEE Vehicular Technology Conference, Stockholm, Sweden, June 1994.

[2] M. Andersin, N. B. Mandayam, and D. Y. Yates. Subspace based estimation of the signal to interference ratio for TDMA cellular systems. Wireless Networks, 4(3), 1998.

[3] J. Blom and F. Gunnarsson. Power Control in Cellular Radio Systems. Licenciate Thesis, Linköpings universitet, Sweden, June 1998. Available at http://www. control.isy.liu.se.

[4] F. Gunnarsson, J. Blom, and F. Gustafsson. Method and system for quality-based power control in cellular communications systems. US Patent Application No. 09/031,635, 1998.

[5] F. Gunnarsson, J. Blom, and F. Gustafsson. Power control in cellular systems subject to constraints and time delays. In Proc. IEEE Global Telecommunications Conference, Sydney, Australia, November 1998.

[6] E.L. Lehmann. Theory of Point Estimation. Statistical/Probability series. Wadsworth \& Brooks/Cole, 1991.

[7] S. C. Schwartz and Y. S. Yeh. On the distribution function and moments of power sums with log-normal components. The Bell System Technical Journal, 61(7), 1982.

[8] G. L. Stüber. Principles of Mobile Communication. Kluwer Academic Publisher, Boston, MA, USA, 1996.

[9] M. Türkboylari and G.L. Stüber. An efficient algorithm for estimating the signal-to-interference ratio in TDMA cellular systems. IEEE Transactions on Communications, 46(6), 1998.

[10] J. Zander. Performance of optimum transmitter power control in cellular radio systems. IEEE Transactions on Vehicular Technology, 41(1), February 1992 . 